\title{
Polyvinylpyrrolidone-Sodium Hyaluronate Gel
}

National Cancer Institute

\section{Source}

National Cancer Institute. Polyvinylpyrrolidone-Sodium Hyaluronate Gel. NCI Thesaurus.

Code C61593.

An oral bioadherent gel containing polyvinylpyrrolidone (PVP) and sodium hyaluronate with muco-protective activity. Upon oral application, this gel adheres to the mucosal surface of the mouth and throat, forming a thin layer that acts as a barrier to protect exposed and sensitized nerve endings from the painful stimuli associated with eating, drinking, and talking. 